Voix et Images

volxetimages

\title{
Bibliographie de Michel van Schendel
}

Roger Chamberland et Pascale Noizet

Volume 11, numéro 2 (32), hiver 1986

Michel van Schendel

URI : https://id.erudit.org/iderudit/200557ar

DOI : https://doi.org/10.7202/200557ar

Aller au sommaire du numéro

\section{Éditeur(s)}

Université du Québec à Montréal

\section{ISSN}

0318-9201 (imprimé)

1705-933X (numérique)

Découvrir la revue

\section{Citer ce document}

Chamberland, R. \& Noizet, P. (1986). Bibliographie de Michel van Schendel.

Voix et Images, 11(2), 256-261. https://doi.org/10.7202/200557ar d'utilisation que vous pouvez consulter en ligne.

https://apropos.erudit.org/fr/usagers/politique-dutilisation/ 
14. Québec, PUL, vol. V, no I-2, janvier-août 1964, p. 153-165.

15. Op. cit.

16. Oeuvres choisies, Paris, Éd. sociales, 1953, p. 290-295.

17. “Idéologie et appareils idéologiques d'état», La Pensée no 151, juin 1970, p. 14.

18. "Appareils et institution (littéraire)». Discours d'ouverture du colloque Discours et histoire, Montréal, 1980.

19. Dialectiques, no 8, Paris, 1975.

20. Les Cahiers de l'ISSH, no 5, août 1976, p. 149-169.

21. Le Roman de Balzac, Montréal, Didier, 1980, p. 195-211.

22. "Mises au point et perspectives à propos de l'analyse automatique du discours". Langages 37, p. 7-30. Paris, Larousse, 1975.

23. Balzac - Sémiotique du personnage romanesque: l'exemple d'Eugénie Grandet, Montréal-Paris, PUM/Didier, 1980.

\title{
Bibliographie $^{1}$ de Michel van Schendel
}

\author{
par Roger Chamberland, Université Laval \\ et Pascale Noizet, Université du Québec à Montréal
}

\section{I - CEUVRES}

Poèmes de l'Amérique étrangère, Montréal, les Éditions de l'Hexagone, (1958), 46 p. reproduit dans De l'oeil et de l'écoute. Poèmes 1956-1976, 247 p. (v. p. 19-51).

Variations sur la pierre, Montréal, Éditions de l'Hexagone, (1964), $46 \mathrm{p}$.

Veiller ne plus veiller; suite pour une grève: poème daté, 17 septembre 1976 - 30 avril 1977.

Saint-Lambert, Éditions du Noroit; ( 1978), 91 p.

De l'ceil et de l'écoute: poèmes 1956-1976, Montréal, l'Hexagone, (1980), 247 p.

Autres. Autrement, Montréal, l'Hexagone, (1983), 100 p.

\section{II - CHAPITRES DE VOLUMES}

"Tendances de la poésie canadienne-française», La Poésie et nous, Montréal, les Éditions de l'Hexagone, (1958), 93 p.

"L'Amour dans la littérature canadienne-française", dans (en collaboration), Littérature et société canadienne-française, Québec, PUL, (1964), 272 p. (v. p. 153-165).

"Le Rôle de l'idéologie dans la poésie canadienne-française", les Juifs et la communauté française, Montréal, les Éditions du jour, Coll. les Idées du jour, (1965), 136 p. (v. p. 115-131).

"L'Apprivoisement du vertige ou la recherche des nouvelles traditions. Poésie québécoise 19601965 ", Livres et auteurs canadiens, (1965), p. 13-22.

"Poésie et précision», "Cette poésie qu'on lit si peu...., «Poésie vivante», Présence de la critique, Textes choisis par Gilles Marcotte, Ottawa, HMH, (1966), 249 p. (v. p. 195-207).

"La Maladie infantile du Québec", dans (en collaboration), les Québécois, préface de Jacques Berque, Montréal et Paris, Librairie François Maspéro et Revie Parti pris, (1967), 295 p. (v. p. 19-38).

1. Michel Van Schendel a beaucoup écrit, tant en Europe qu'au Québec. La présente bibliographie porte essentiellement sur les textes parus au Québec. Sont exclues pour des raisons d'espace d'autres séries radiophoniques et les séries télévisuelles versées aux archives de la revue. 
"Ducharme l'inquiétant", Littérature canadienne-française, Conférences J.-A. de Sève, Montréal, les Presses de l'Université de Montréal, (1969), 346 p. (v. p. 216-234).

"Du refus global à une poésie de l'appartenance», en participation avec Pierre de Grandpré, Histoire de la littérature française du Québec, T. III, Montréal, Éditions Beauchemin, (1969), 407 p. (v. p. 203-287).

«Manuels. Cênsure. Privilèges», Brèches, Analyse/Fiction, (printemps-été 1976), p. 7-44.

«Modes idéologiques du discours poétique», Littérature et idéologie, Québec, P. U.L., (1976), 339 p. (v. p. 149-169).

«Appareil et Institution (littéraire)», Recherches et Théories, Montréal, PUQ, (1979), p. 1-14 no 19).

"Analyse d'une 'composition'», le Roman de Balzac: recherches critiques, méthodes, lectures, Montréal, Éditions Didier, (1980), p. 195-211.

«Nous: qu'est-ce que 'nous'? ", le Dialogue, Montréal, Ėditions Didier, coll. 3 L, (1985), 182 p. (v. p. 63-76).

"Sentiers critiques», Sédiments, Montréal, HMH, sous la direction de Georges Leroux et Michel van Schendel, 1986.

\section{III - ARTICles DE PÉRIODIQUES}

"Cinéma des désirs", Vie des arts, no 12 (automne 1958), p. 19-26.

"Langage, poésie et engagement», Liberté, vol. 1, no 1, (janvier-février 1959), p. 11-21.

«Chroniques. La littérature cańadienne. La poésie cherche à bercer l'âme alors qu'elle devrait pétrir les choses", Liberté, vol. III, no 1 (janvier-février 1961), p. 445-451.

"Yves Bonnefoy ou la mort vivante", Liberté, vol. III, nos 15-16 (mai-août 1961), p. 654-657.

"L'espèce de conscience qu'il fallait», Situations, $4^{e}$ année, no 2 (juillet 1962), p. 20-25.

"Le Scandale Sidbec, c'est le scandale Lesage», Cité libre, XVe année, no 78 (juillet 1965), p. 1-4. "L'Essoufflement libéral ou l'apologue de la puce», Socialisme, no 7 (janvier 1966), p. 31-37.

"Le Mur", la Barre du jour, vol. I, no 6 (janvier-février 1966), p. 2-4.

«Le Québec à l'heure américaine», Socialisme, no 8 (mai 1966), p. 7-28.

"Fusion et confusion", Socialisme, nos 9-10 (octobre-décembre 1966), p. 20-25.

"Ottawa est complice du massacre", Socialisme, nos 12-13 (avril-mai-juin 1967), p. 3-14.

"Pas de socialisme sans lutte contre l'impérialisme américain", Socialisme, nos 12-13 (avril-maijuin 1967), p. 94-106.

"La Part de l'enseignement de la littérature dans l'acquisition d'une culture littéraire", Liberté, $\# 57$, vol. X, nos 3-4 (mai-juin 1968), p. 57-66.

"Émiettements...», la Barre du jour, no 14 (juin-juillet 1968), p. 32-40.

"Pour une théorie et un programme socialistes au Québec. Portée des événements internationaux de 1968 ", Socialisme, no 15 (octobre-novembre-décembre 1968), p. 3-18.

"Pour une théorie du socialisme québécois (II)), Socialisme, no 17 (avril-mai-juin 1969), p. 7-26.

"Les Ecrivains, la littérature et les mass-média", Liberté, vol. II, nos 3-4 (mai-juin-juillet 1969), p. 74-86.

"La conférence hémisphérique est un modèle à suivre", Socialisme, no 16 (janvier-février-mars 1969), p. 7-10.

"Conditions d'une poésie critique», Socialisme québécois, no 20 (avril-juin 1970), p. 58-71.

«Impérialisme et classe ouvrière au Québec (esquisse d'analyse)», Socialisme québécois, nos 21-22 (1971), p. 156-209.

"Notes: dépendances et autonomies politiques de la classe ouvrière», Contradictions, Bruxelles, no 3 (janvier-juin 1973), p. 53-102.

"La Balle des mots", Liberté, \#97-98, vol. 17, nos 1-2 (janvier-avril 1975), p. 100-110, 119-120 (reproduit dans Change. Souverain Québec, mars 1977, p. 210-220).

«Ellipse et valeur, analyse d'une aporie saussurienne», Dialectiques, Paris, no 8 (printemps 1975), p. 111-118. 
"Cette écriture», Voix et images, vol. IV, no 1 (septembre 1978), p. 134-139 [reproduit sous le titre "De l'écoute", dans De l'ceil et de l'écoute. Poèmes 1956-1976, 247 p. (v. p. 227-238)].

«Je le dis à ces amis», Estuaire, no 16 (juin 1980), p. 89-92.

«Balzac: de l'œuvre au texte», University of Toronto Quarterly, vol, 51, no 1 (Fall 1981), p. 112-124.

"De récession et en récession: synchronisation des cycles", Interventions, no 6 (hiver 1981), p. 24-33.

"Cammin", Estuaire, no 25 (automne 1982), p. 42-58.

"Refus global, or the Formula and History", Yale French Studies, New Haven, no 65 (1983), p. $53-73$.

\section{IV - TEXTES JOURNALISTIQUES}

"Entre le poète et le public, se dresse un mur", Le Devoir, 11 mars 1961.

"Un courant nouveau en poésie: la saisie de notre univers physique», Le Devoir, 25 février 1961.

Articles parus dans Le Nouveau journal: du 16 septembre 1961 au 19 mai 1962.

1. "Le drame d'une province que la guerre fait l'arbitre de l'unité congolaise»; 2. «M. 'H' a-t-il été tué délibérément?»; 3. "L'ONU dans une impasse»; 4. "Un neutralisme actif comme celui du Ghana»; 5. "Rusk et Gromyko tentent de trouver une solution provisoire»; 6. "Une consigne de sagesse: procéder avec prudence"; 7. "Après le discours Gromyko: la diplomatie reprend ses droits à l'ONU»; 8. «Le neutralisme devient l'idée-force"; 9. «Le peuple américain se livre à la guerre du risque»; 10. "Marché commun: l'Europe unie..."; 11. “Obscure Albanie terre de tyrans"; 12. "Berlin. Qui porte la responsabilité des 4 crises d'après-guerre?»; 13, «1961: les griffes de la paix»; 14. "La bête du nazisme respire encore...»; 15. "Contre les tueurs de l'OAS, des Français, déjà, commencent à sortir les fusils de la résistance»; 16. "Entre 'K' et Tito une ombre chinoise".

Articles parus dans La Presse en 1963 dans la série . “l'Investissement international. Interdépendance des pauvres, profit des riches.»

1. "Quand l'investissement obéit à deux poids, deux mesures"; 2. "Le commerce mondial réduit sans cesse la part du pauvre"; 3. "Comment le libre échange peut cloisonner les échanges"; 4. "Comment les riches entendent raffermir leur contrôle»; 5. «Principale question politique des dix prochaines années»; 6. "Les Mexicains éprouvent les caprices du capital étranger"; 7. "Les leçons que le Mexique peut donner au Québec".

"En Belgique, Wallons et Flamands à couteaux tirés», Le Magazine Maclean, (novembre 1962), p. 20/70-79.

\section{V - TEXTES RADIOPHONIQUES}

Série diffusée à Radio-Canada dans le cadre de l'émission «Chacun sa vérité» 1956-1957-1958 sur: 1. John Keats; 2. Frederico Garcia Lorca; 3. Diderot; 4. Voltaire; 5. André Malraux; 6. Romain Rolland.

Série diffusée à Radio Canada dans le cadre de l'émission . «Poésie étrangère», 1957, sur: 1. Walt Whitman; 2. Aimé Césaire; 3.' Rafael Alberti; 4. Poésie chinoise.

Série diffusée à Radio-Canada dans le cadre de l'émission “Revue des arts et des lettres», de 1956 à 1960, sur: 1. Jules Romains; 2. Philippe Hériat; 3. Claude Mauriac et Laurent La Praye; 4. Henry Miller; 5. Pamela Moore; 6. Hervé Bazin; 7. Colette; 8. Robert Elie; 9. Jean-Charles Harvey; 10. Jean Filiatrault et Jean Pellerin; 11. Alain Grandbois; 12. Jules Gobeil et Gérard Bessette; 13. Pierre Trottier et Alain Horic; 14. Paul Guimard; 15. Michel Butor; 16. Loys Masson; 17. Roger Vailland; 18. Albert Camus; 19. Paul Vialar; 20. Madeleine Ferron; 21. Yves Bonnefoy; etc.

Série d'histoire et de littérature diffusée à Radio-Canada dans le cadre de l'émission aIdes et calandes du roman au Canada», 1957, sur: 1. Le roman: "Honni soit qui bien y pense»; 2. Narrateurs et romanciers; 3. Naissance d'une respiration; 4. La ville quotidienne; 5. De la guerre à la paix; 6 . Aspects et figures de la poésie canadienne-anglaise; 7. Demain, les civilisés. 
Série diffusée à Radio-Canada dans le cadre de l'émission "Morceaux d'anthologie", 1957-1958, sur: 1. Félix-Antoine Savard; 2. Ringuet; 3. Berthelot Brunet; 4. Jean Simard; 5. Alain Grandbois; 6 . André Langevin.

Série diffusée à Radio-Canada dans le cadre de l’émission «Partage du matin", 1958-1959, sur les tâches féminines: 1 . La ravaudeuse, la peineuse; 2 . La lavandière; 3 . La cuisinière; 4. Les fonctions domestiques; 5 . Le travail des femmes. La tisserande; 6 . De la résistance des femmes et de la notion de courtoisie; 7 . L'intouchable, la dame de haut parage; 8 . Une mère, quand elle est jeune... Une mère. quand elle vieillit...

Poèmes radiophoniques diffusés à Radio-Canada dans le cadre de l'émission "Images du Canada ". Avril et mai 1959: 1. Sons et saisons. Été; 2. Sons et saisons. Automne; 3. Sons et saisons. Hiver.

Série diffusée à Radio-Canada dans le cadre de l'émission «Grandes correspondances", 19591960. sur: 1. Dostoievski; 2. Vincent Van Gogh; 3. Napoléon; 4. Marie-Thérèse; 5. Holderlin; 6. Claude Debussy; 7. Colette; 8. Apollinaire.

Série diffusée à Radio-Canada dans le cadre de l'émission "Personnages en liberté», 1960, sur: 1. Le Christ; 2. Julien Sorel; 3. Hamlet; 4. Prométhée; 5. L'étranger (Albert Camus); 6. Don Juan; 7. Celui qui avait faim (La faim de Knut Hamsun); 8. Faust; 9. Electre; 10. Raskolnikov; 11. Caligula (Caligula d'Albert Camus); 12. Rastignac; etc.

Série diffusée à Radio-Canada dans le cadre de l'émission . "Des livres et des hommes", 1967-1968, sur: 1. Fernand Ouellette; 2. Gatien Lapointe; 3. Yves Préfontaine; 4. Réjean Ducharme; 5. Lorenzo Morin; 6. Paul Chamberland; 7. La Barre du jour; 8. Les éditions de l'Estérel; 9. Serge Legagneur et Gemma Tremblay; etc.

\section{VI - TRADUCTIONS}

"Aspects et figures de la poésie canadienne-anglaise", Cahiers radiophoniques, vol. I, no 1 , (1958). p.81-86.

Le Nouveau parti démocratique, Montréal, Éditions du jour, 1960.

Une révolution tranquille, Montréal, HMH, 1965, $159 \mathrm{p}$.

Le "Canadien Pacifique», Montréal, PUM, 1968, $206 \mathrm{p}$.

Histoire du syndicalisme au Canada et au Québec, Montréal, Éditions Parti pris, 1974-1976, $506 \mathrm{p}$.

\section{VII - ÉTUDES Ã CONSULTER (sur l'œuvre en général)}

HAMEL, Réginald, John HARE et Paul WYCZYNSKI, Dictionnaire pratique des auteurs québécois, Montréal, Fides, (1976), p. 677-678.

MAILHOT, Laurent et Pierre NEPVEU, la Poésie québécoise des origines à nos jours. Anthologie, Québec/Montréal, PUQ/les Éditions de l'Hexagone, (1980), p. 393-397.

UNION DES ÉCRIVAINS QUÉBÉCOIS. Dictionnaire des écrivains québécois contemporains, Montréal, Québec/Ampérique, (1983), p. 387-388.

\section{VIII - ÉTUDES À CONSULTER (sur les cuvres en particulier)}

\section{Poèmes de l'A mérique étrangère:}

ANONYME, "Poèmes de l'Amérique étrangère". Bulletin du Cercle juif, $5^{\mathrm{c}}$ année. no 43 (mars 1959), p. 4.

BROSSARD, Nicole, "Van Schendel», le Quartier latin (le Nouveau Cahier), vol. XLIX, no 7 (6 octobre 1966), p. 4-5.

DU HAMEL, Roger, "Courrier des lettres. Nos jeunes écrivains conservent intacte leur foi en la poésie [...] Poèmes de l'Amérique étrangère de Michel Van Schendelm, la Patrie (du dimanche), vol. XXV, no 12 (22 mars 1959), p. 34.

GRANDPRÉ, Pierre de, «la Vie des lettres. Un accent neuf en poésie canadienne. Poèmes de l'Amérique étrangère"', le Devoir, vol. L. no 31 ( 7 février 1959), p. 11.

-, "Du Refus glohal à une poésie de l'appartenance: les post-surréalistes». Histoire de la littérature française du Québec, tome 111 ; Ia Poésie (1945 à nos jours). Montréal, Librairie Beauchemin limitée. 1969. 407 p. (v. p. 266-269). 
MARCOTTE, Gilles, "Chronique. Recherche des signes», Liberté 59, vol. 1, no 1 (janvier-février 1959), p. 45-49 (v.p. 47-48).

- "Poésie pour nommer: Michel Van Schendel", le Temps des poètes. Description critique de la poésie actuelle au Canada français, (Mont-réal), HMH, (1969), 247[1] p. (v. p. 166-168).

PARADIS, Suzanne, "Poèmes de l'Amérique étrangère", Dictionnaire des ceuvres littéraires du Québec, t. III, 1940-1959, Montréal, Fides, (1982), p. 771-773.

SYLVESTRE, Guy, "Letters in Canada: 1958. Livres en français. La poésie», University of Toronto Quarterly, vol. XXV, (1959), p. 444-448 (v. p. 451).

Variations sur la pierre:

[ANONYME], «les Livres. Variations sur la pierre par Michel Van Schendel [...]", Bulletin du cercle juif, vol. 10, no 96 (septembre-octobre 1964), p. 3.

BASILE, Jean, «la Semaine littéraire. À l'occasion du lancement de Variations sur la pierre. Michel Van Schendel: 'Pour moi, il est aussi important d'écrire un article qu'un poème...'", le Devoir, vol. LV, no 221 (19 septembre 1964), p. 15.

BEAULIEU, Michel, "Variations sur la pierre", le Quartier latin, vol. XLVIII, no 8 (8 octobre 1964), p. 1.

BROSSARD, Nicole, "Van Schendel», le Quartier latin (le nouveau Cahier), vol. XLIX, no 7 (6 octobre 1966), p. 4-5.

CHAMBERLAND, Roger, "Variations sur la pierre", Dictionnaire des œeuvres littéraires du Québec, t. IV. 1960-1969, Montréal, Fides, (1984), p. 937-938.

GRANDPRE, Pierre de (éditeur), «Du Refus global à une poésie de l'appartenance: les Postsurréalistes", Histoire de la littérature française du Québec, t. III; la Poésie (de 1945 à nos jours). Montréal, Librairie Beauchemin limitée, 1969, 407 p. (v. p. 266-269).

LAROCHE, Maximilien, "Variations sur la pierre de Michel Van Schendel», Livres et auteurs canadiens, 1964, p. 68-69.

MARCOTTE, Gilles, «Littérature. Michel Van Schendel», la Presse (supplément), vol. LXXXI, no 18 (23 janvier 1965), p. 6.

-, "Poésie pour nommer: Michel van Schendel ", le Temps des poètes: Description critique de la poésie actuelle au Canada français, Montréal, HMH, (1969), 247[1] p. (v. p. 166-168).

MELANÇON, André, «Notices bibliographiques. Littérature canadienne. Littérature. Schendel (Michel van) Variations sur la pierre [...]", Lectures, vol. 12, no 1 (septembre [965), p. 12-13.

PELOSSE, Cécile, «la Recherche du pays dans la poésie québécoise de 1945 à 1970 ». Thèse de doctorat, Montréal, Université de Montréal, 1974, vi, $473 \mathrm{f}$.

POULIN, Marc, «Poésie. Variations sur la poésie», le Quartier latin (supplément), vol. XLVII. no 29 (21 janvier 1965). p. 4.

PRÉFONTAINE, Yves, “Poésie pas morte!", Maintenant, no 42 (juin 1965), p. 211.

SYLVESTRE, Guy, "les Livres. Poésie canadienne. Variations sur la pierre de Michel van Schendel", le Devoir, vol. LV, no 227 (26 septembre 1964), p. 16.

-, "Livres en français. La poésie», University of Toronto Quarterly, vol. XXXIV, no 4 (July 1965), p. 464-475 (v. p. 472)

THÉBERGE, Jean-Yves, «la Pierre et le Québec en poésie», le Canada français, vol. CV, no 20 (8 octobre 1964), p. 26.

VACHON, André, "Chronique des lettres. Tardif début de saison", Relations, no 288 (décembre 1964), p. 362 [reproduit dans Québec 65, vol. 2 (mai 1965), p. 93-95].

Veiller ne plus veiller:

BOURASSA, André-G.: "Entre l'espace et le temps. [Veiller ne plus veiller]", Lettres québécoises, no 14 (avril-mai 1979), p. 20-21.

DUPRE, Louise, "[Figure de grève. Veiller ne plus veiller]", Voix et images, vol. V, no 3 (printemps 1980), p. 597-598.

PARADIS, Suzanne, "Veiller ne plus veiller", le Devoir, vol. LXX, no 112 (14 mai 1979), p. 23. 
ROYER, Jean, "Michel Van Schendel. Ce délire au-delà de la fátigue ", le Devoir, vol. LXX, no 110 (12 mai 1979), p. 19-20 [reproduit dans Écrivains contemporains. Entretiens 2: 1977-1980, (Montréal), l'Hexagone, (1983), p. 133-139].

\section{De l'aeil et de l'écoute:}

ANONYME, 1980 Governor General's Literary awards [for De l'oeil et de l'écoute]". Parallelogramme, vol. 6, no 5 (May-June 1981), p. 33.

— «Governor General's award winners", Quill and Quire, vol. 47, no 6 (June 198I), p. 19.

- «Le Poète Van Schendel, prix du gouverneur général». Réseau, vol. 13, no 1 (septembre 1981), p. 6.

— «rix du Gouverneur général 1981 [pour de l'Oeil et de l'écoute]". Lettres québécoises, no 22 (été 1981), p. 71.

BAYARD, Caroline. «Livres en français, la poésie". University of Toronto Quarterly. vol. L, no 4 (summer 1981), p. 48-49.

BEAUSOLEIL, Claude, «Michel Van Schendel. L'Intelligence du réel», le Devoir, vol. LXXI, no 297 (27 décembre 1980), p. 16.

BOUVIER, Luc, “De l'ceil et de l'écoute», Livres êt auteurs québécois, 1980, p. 141-143.

BROCHU, André, "Rétrospectives et prospectives [De l'aeil et de l'écoute]", Voix et images, vol. VII, no 3 (printemps 1982), p. 584.

PARADIS, Suzanne, "De l'œil et de l'écoute», Estuaire, no 22 (hiver 1981), p. 88-89.

CORRIVEAU. Hugues, "Des voix plurielles [ De l'aeil et de l'écoute]", Spirale, no 12 (octobre 1980), p. 12.

GIGUĖRE, Richard, "L'Emploi d'écrire, [De l'ceil et de l'écoute]", Lettres québécoises, no 19 (automne 1980), p. 32-33.

\section{Autres, autrement:}

BROCHU, André, «Poésie. Écriture en locomobile», Voix et images, vol. X, no 1 (automne 1984), p. 151-152.

YERGEAU, Robert, «la Poésie dans tous ses états: Langevin, Vanier, Geoffroy, Van Schendel, Cloutier", Lettres québécoises, no 34 (été 1984), p. 30-34.

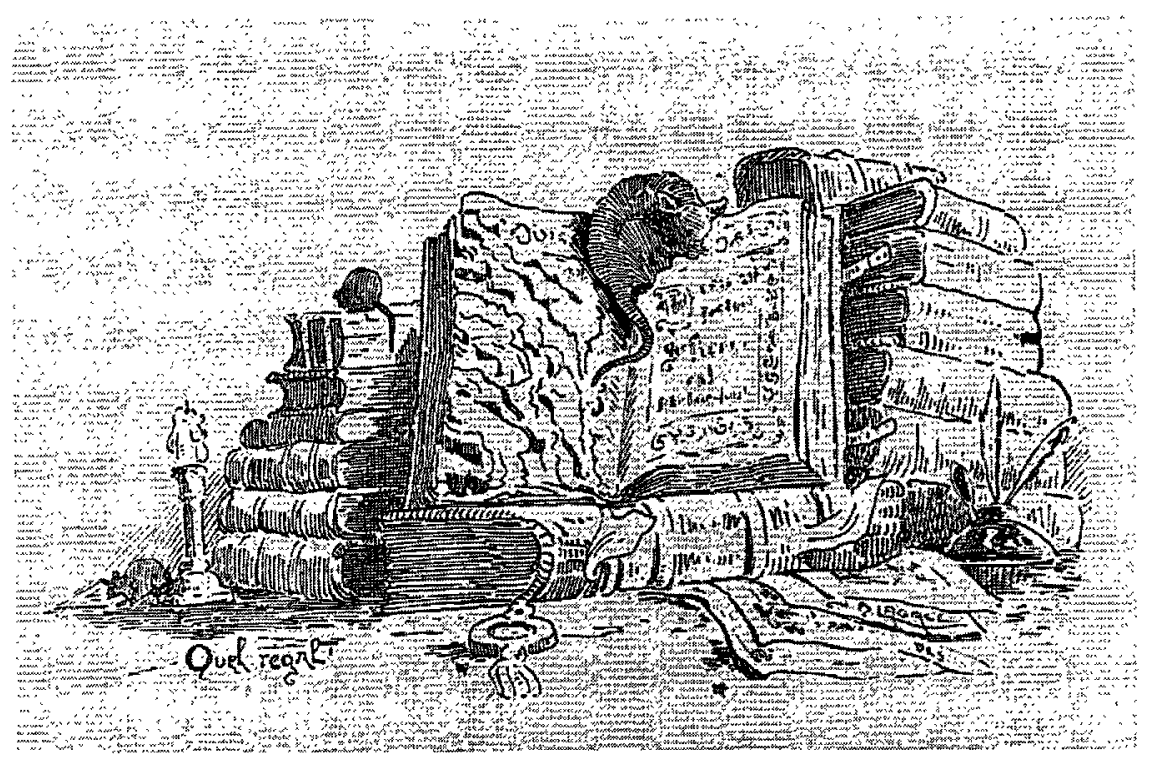

